\title{
Aromatasehemmer bei Brustkrebs: Therapie auf 10 Jahre verlängern?
}

Die Therapie mit einem Aromataseinhibitor (Al) über 5 Jahre von Anfang an oder nach einer Tamoxifen-Therapie ist die Behandlung der Wahl für postmenopausale Frauen mit Hormonrezeptor-positivem frühem Brustkrebs. Die Gabe des Al für bis zu 10 Jahre könnte das Rezidivrisiko weiter reduzieren.

D as Rezidivrisiko bei Hormonrezeptor (HR)-positivem Brustkrebs bleibt für unbegrenzte Zeit bestehen. Daher nehmen viele Patientinnen einen AI über mehr als 5 Jahre ein. Studiendaten zur Reduktion des Rezidivrisikos lagen bisher aber nur über eine Behandlungsdauer von bis zu 5 Jahren vor.

Die doppelblinde, placebokontrollierte Phase-III-Studie MA.17R der North American Breast Cancer Group (NABCG) liefert nun 10-Jahres-Daten. 1.918 postmenopausale Patientinnen hatten zuvor bereits 4,5-6 Jahre lang eine adjuvante AI-Therapie bekommen, meist nach Tamoxifen. In MA.17R erhielten sie für weitere 5 Jahre randomisiert entweder 2,5 mg/Tag Letrozol oder Placebo. Primärer Endpunkt war das krankheitsfreie Überleben (DFS).

Nach median 6,3 Jahren lag die 5-Jahres-Rate für das DFS bei $95 \%$ unter Letrozol und bei $91 \%$ unter Placebo (Hazard Ratio [HR] 0,66; $\mathrm{p}=0,01)$. Die Rate für das 5-Jahres-Gesamtüberleben betrug 93 im Vergleich zu $94 \%$ (HR 0,97; $\mathrm{p}=0,83)$. Die jährliche Inzidenzrate kontralateraler Tumoren lag bei 0,21 versus $0,49 \%$ (HR 0,$42 ; p=0,007)$. In allen Subgruppen war der positive Einfluss von Letrozol nachweisbar.

Mit Ausnahme von Effekten auf die Knochen war die Inzidenz von Toxizitäten in beiden Gruppen vergleichbar. Knochenschmerzen, Knochenbrüche und Osteoporose waren unter Letrozol häufiger als unter Placebo. Einige Frauen brachen die Therapie aufgrund der Toxizitäten ab (5,4 vs. 3,7\%).

In den Summenscores zur Lebensqualität, ermittelt mit dem SF-36-Fragebogen, fand sich kein signifikanter Unterschied zwischen beiden Gruppen. Auch in den meisten Subskalen gab es keine signifikanten Unterschiede.

Fazit: Postmenopausale Frauen mit Brustkrebs profitierten von der Verlän- gerung der adjuvanten Therapie mit einem Aromataseinhibitor auf bis zu 10 Jahre. Damit verbesserten sich ihr DFS und die Inzidenz kontralateraler Brusttumoren signifikant im Vergleich zu Placebo. Das Gesamtüberleben wurde allerdings nicht verbessert. Judith Neumaier

Goss PE et al. Extending Aromatase-Inhibitor Adjuvant Therapy to 10 Years. N Engl J Med. 2016;375(3):209-19.

\section{Kommentar von Prof. Jackisch:}

"I was the one who advised you not to do this trial in the first place" war der Kommentar von lan Smith vom Royal Marsden Hospital in London auf der Jahrestagung der American Society of Clinical Oncology (ASCO) 2016 in Chicago, nachdem Paul Goss, Boston, MA/USA, die MA.17R-Daten präsentiert hatte. Die MA.17R-Studie gibt Aufschluss über die Durchführbarkeit und Akzeptanz einer verlängerten adjuvanten, zielgerichteten Behandlung, nämlich der endokrinen Therapie beim Mammakarzinom. Sie ist eine der effektivsten adjuvanten Therapieformen, erfordert aber auch eine große Adhärenz und Compliance der Patientinnen. Frauen mit frühem endokrin responsivem Mammakarzinom (Östrogen- und/oder Progesteronrezeptor-positiv) wurden nach 5-jähriger Tamoxifentherapie, bei Fehlen eines Rückfalls, für 4,5-6 Jahre mit einem Al behandelt und danach für weitere 5 Jahre randomisiert mit Letrozol oder Placebo.

Die Resultate zeigten eine signifikante Verbesserung des DFS durch eine zusätzliche 5-jährige Therapie mit Letrozol. Eine signifikant höhere Rate für das 5-JahresGesamtüberleben konnte jedoch nicht nachgewiesen werden. Ein Grund für den fehlenden Überlebensvorteil ist sicherlich der hohe Anteil kontralateraler Zweitkarzinome an den dokumentierten Ereignissen - die um 58\% gesenkt wurden (HR 0,42; $p=0,007)$. Somit wurde zwar das DFS verbessert. Dies spiegelt sich aber nicht in einer Verlängerung des Überlebens wider.
Hinsichtlich der Nebenwirkungen wurde nach zusätzlicher 5-jähriger Behandlung mit Letrozol eine Verdoppelung der neu aufgetretenen Osteoporosen (11 vs. $6 \%$; $p<0,0001)$ und eine Erhöhung der Frakturrate von $9 \%$ auf $14 \%(p=0,001)$ festgestellt. Ob wir unseren Patientinnen nun zukünftig zu einer 15-jährigen antihormonellen Therapie raten sollten, muss individuell abhängig von der bisherigen Therapieverträglichkeit und dem Rückfallrisiko entschieden werden. Der Wert dieser Analyse bleibt unbenommen. Dennoch kann aus den Daten, auch unter Einschluss der sehr guten Lebensqualität, keine generelle Empfehlung für eine 10-jährige endokrine Therapie bei allen Patientinnen abgeleitet werden. Für die Indikationsstellung zu einer erweiterten adjuvanten endokrinen Therapie beim endokrin responsiven Mammakarzinom können die Daten der EBCTCG-Analyse mit 46.138 Patientinnen hilfreich sein. Sie wurden ebenfalls bei der ASCO-Jahrestagung 2016 vorgestellt [Pan $\mathrm{H}$ et al. J Clin Oncol. 2016; 34(suppl):Abstr 505]. Hier wurde eindrucksvoll belegt, dass auch

\section{"Die Indikation zur erweiterten adjuvanten Therapie sollte individuell gestellt werden."}

heute Tumorgröße, Nodalstatus und das nukleäre Grading eine große Rolle für das Rückfallrisiko spielen.

Die Indikation für eine erweiterte adjuvante Therapie (Jahr 5-10 nach Diagnosestellung) sollte im individuellen Gespräch mit der Patientin gestellt werden. Die Ergebnisse der MA.17R-Studie umzusetzen (Jahr 10-15), stellt dann aber sehr hohe Anforderungen an die Therapiecompliance. Außerdem ist dabei eine umsichtige Reevaluation der Knochengesundheit erforderlich, um eine unnötige Morbiditätserhöhung zu vermeiden.

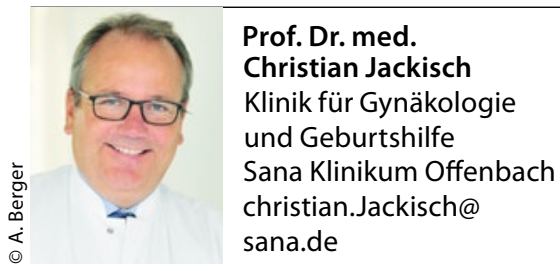

